\title{
Stability of cross-shaped electron beam in a vacuum magnetic sensor with a p-Si field emitter tip
}

Koji Uemura ${ }^{1}$, and Takashi Suemasu ${ }^{2}$

${ }^{1}$ YASKAWA Electric Corporation, Kitakyushu, Fukuoka 806-0004, Japan

${ }^{2}$ Institute of Applied Physics, University of Tsukuba, Tsukuba, Ibaraki 305-8573, Japan

\begin{abstract}
The stability of the cross-shaped electron beam (EB) in a vacuum magnetic sensor with a vertical p-type Si field emitter tip was investigated. The magnetic flux parallel to the $\mathrm{EB}, B_{z}$, was detected by rotational displacements $\Delta \Phi$ of the cross-shaped EB between the anodes, and sensed as a differential anode current normalized by total current $\Delta I_{\mathrm{a}} / I_{\mathrm{a}}$. The fluctuation in $\Delta \Phi$ was within $1 \%$ over a duration of $90 \mathrm{~s}$ at $B_{\mathrm{z}}=3.0 \mathrm{mT}$. The magnetic flux normal to the $\mathrm{EB}, B_{x}$, was detected by the translational displacement $\Delta L_{y}$ of the cross-shaped EB along the $y$ direction, and sensed as $\Delta I_{\mathrm{a}} / I_{\mathrm{a}}$. The fluctuation in $\Delta L_{y}$ was also within $1 \%$ at $B_{x}=3.0 \mathrm{mT}$. These results demonstrate that this sensor with a vertical $\mathrm{p}$-Si emitter tip using the cross-shaped EB is able to detect the magnetic flux in an arbitrary direction with high sensitivity and stability.
\end{abstract}

Keywords vacuum microelectronics, field emitter, vacuum magnetic sensor, focusing election beam, current stability

Corresponding author:

Takashi Suemasu

Institute of Applied Physics, University of Tsukuba

Tsukuba, Ibaraki 305-8573, Japan

suemasu@bk.tsukuba.ac.jp 


\section{Introduction}

Recently, vacuum microelectronics devices exploiting ballistic electrons in vacuum have attracted attention since the realization of the micrometer-sized field emission cathode by Spindt and colleagues. ${ }^{1,2)}$ Vacuum microelectronics devices are called field emitters because the emitters, formed by fine processing technology, generate an emission field of electrons. Much work have been devoted to metal electrodes as field emitters. ${ }^{3-6)}$ Such devices have been studied in a wide range of applications such as displays, ${ }^{2,7,8)}$ microwave waveguides, ${ }^{9,10)}$ free electron lasers, ${ }^{11)}$ electron microscopes, ${ }^{12,13)}$ vacuum gages, photo sensors, ${ }^{14-18)}$ and reliable uniform and high current emissions. ${ }^{19,20)}$

The present study focuses on magnetic sensors using features of vacuum microelectronics devices, particularly those with separate electrodes. ${ }^{21)}$ At first, magnetic sensors, wherein the transport of carriers in the semiconductor is exploited, were studied to meet size-reduction requirements. ${ }^{22-24)}$ However, these devices presented difficulties regarding performance improvements such as enhancing sensitivity. Such difficulties were mostly caused by the limited mobility of carriers in the semiconductors. For this reason, the research focus gradually shifted to magnetic sensors using the transport of electrons in vacuum. Vacuum magnetic sensors (VMS) having a horizontal field emitter array (FEA) made of tungsten $(\mathrm{W})^{25-27)}$ demonstrated their superiority over semiconductor-based magnetic sensors in regard to robustness and sensitivity. ${ }^{28)}$ The characteristics showed a sensitivity as high as $1,000 \% / \mathrm{T}$ (T being the unit of magnetic flux density $B$ ). This sensitivity is about one order of magnitude higher than that of semiconductor-based sensors. However, such VMSs are sensitive only to $B$ fields normal to the FEA. We therefore proposed a distinctive three-dimensional (3D) VMS concept and fabricated vertical emitter tips that generate a cross-shaped electron beam (EB) for the $3 \mathrm{D}-\mathrm{VMS}{ }^{29)}$

Scanning electron microscopy (SEM) image and the cross sectional schematic of the proposed VMS are shown in Figs. 1(a) and (b), respectively. The VMS using a vertical emitter consists of a single cone-type $\mathrm{Si}$ emitter tip in the center, a cross-shaped gate electrode, four in-plane focusing lens electrodes, and anode plates as shown in Fig. 1(c). The anode is divided into eight parts in a radial pattern and positively biased to collect electrons. The feature of our VMS is the cross-shaped EB produced by the cross-shaped electrode in Fig. 1(a). EB emitted from the emitter tip experiences the electric filed caused by the focusing lens electrodes, and forms cross-shaped EB patterns as schematically shown in Fig. 1(d). By using such 
cross-shaped EB and separated anodes, the proposed VMS can detect magnetic flux in an arbitrary direction. Briefly, when the magnetic flux is parallel to the $\mathrm{EB}\left(B_{\mathrm{z}}\right)$, the EB rotates around the $z$ axis, leading to the difference in anode current $\left(\Delta I_{\mathrm{a}} / I_{\mathrm{a}}\right)$ between two neighboring anode electrodes because of the cross-shaped EB. Here, $\Delta I_{\mathrm{a}}$ is the imbalance between the two anode parts we choose, and $I_{\mathrm{a}}$ is the total anode current detected by them. By detecting this difference, we can detect $B_{\mathrm{z}}$. The rotation of EB is caused by the Lorentz force acting on the emitted electrons from the tip because they also have velocity components normal to the tip-anode direction. ${ }^{30)}$ When the magnetic flux is normal to EB, the translational displacement of the cross-shaped EB occurs, inducing $\Delta I_{\mathrm{a}} / I_{\mathrm{a}}$. In any case, we can choose two anode electrodes out of the eight ones, and measure $\Delta I_{\mathrm{a}} / I_{\mathrm{a}}$. Currents due to the field emission of electrons fluctuate in principle because they are based on a quantum effect. However such fluctuations mitigate in the proposed VMS by detecting the imbalance $\Delta I_{\mathrm{a}}$ normalized by the total anode current $I_{\mathrm{a}}$ to suppress the effect of the noise in field emission. The VMS is thus robust against common mode noise. ${ }^{31)}$ We achieved good linearity in detecting currents with the VMS subject to an external field $B$, with a sensitivity of $10,000 \% / \mathrm{T}$ for this structure. ${ }^{31-34)}$ However, we have yet to investigate the current stability of the VMS using Si field emitter tips. In this work, we measured the current stability of p-type Si VMS over a duration of $90 \mathrm{~s}$ when the magnetic flux is parallel to the $\mathrm{EB}\left(B_{\mathrm{z}}\right)$ and normal to the $\mathrm{EB}\left(B_{\mathrm{x}}\right)$.

\section{Experimental methods}

The field emitter tips were fabricated by a conventional Spindt-type production technique ${ }^{35}$ ) using a p-type Si substrate with a resistivity $\rho$ of approximately $30-50 \Omega \mathrm{cm}$. The fabrication process flow including SEM images is shown in Fig. 2. Briefly, a 0.3- $\mu$ m-thick $\mathrm{SiO}_{2}$ layer was first made by thermal oxidization, and a $1-\mu \mathrm{m}$-thick positive photoresist was spin-coated and a $1.5-\mu \mathrm{m}$-diameter disk pattern was made using the photolithography technique on the $\mathrm{Si}$ substrate. The resist pattern was transferred to the $\mathrm{SiO}_{2}$ layer by wet etching with buffered hydrofluoric acid (BHF); the resist was removed afterwards. Reactive ion etching (RIE) was performed with $\mathrm{SF}_{6}$ gas to shape the tip into a cone underneath the $\mathrm{SiO}_{2}$ disk pattern. The surface of the Si substrate was again thermally oxidized to sharpen the emitter tip. Then a $0.2-\mu \mathrm{m}$-thick $\mathrm{Nb}$ layer was deposited by $\mathrm{EB}$ evaporation for the gate plate. The $\mathrm{SiO}_{2}$ disk pattern was lifted off by wet etching with BHF to complete the emitter tips. After this process, the surface of the Si emitters was terminated by $\mathrm{H}$ atoms as described in Ref. 32. Finally, the 
$\mathrm{Nb}$ layer was patterned by RIE to form electrodes for gate and focusing lens in the same plane on the substrate. ${ }^{32)}$

The $\mathrm{SiO}_{2}$ layer underneath the gate electrode (Fig. 2) is approximately $1 \mu \mathrm{m}$ thick. The diameter of the gate opening is about $2 \mu \mathrm{m}$. The cross-shaped gate is $2 \mu \mathrm{m}$ wide and $0.9 \mathrm{~mm}$ long. Each focusing lens electrode is separated from the gate electrode by $2 \mu \mathrm{m}$. Similar emitter tips using an n-type Si substrate with $\rho=1-2 \Omega \mathrm{cm}$ were formed for comparison. The device was set in a vacuum chamber with a pressure below $1.33 \times 10^{-6} \mathrm{~Pa}$ and was connected to a power supply and some measurement equipment. The anode plate was biased to $700 \mathrm{~V}$ and the emission current $I_{\mathrm{a}}\left(=I_{\mathrm{a} 1}+I_{\mathrm{a} 2}\right)$, and the anode-current imbalance $\Delta I_{\mathrm{a}}=\left|I_{\mathrm{a} 1}-I_{\mathrm{a} 2}\right|$ were measured.

\section{Results and discussion}

\subsection{Operation principle of Si field emitter}

We herein explain the operation principle of the n-type and p-type Si emitter tips (Figs. 3 and 4). The distance between the anode plate coated with $\mathrm{ZnO}: \mathrm{Zn}$ phosphors and the emitter tip (d) is about $5 \mathrm{~mm}$, and is used for beam profile monitoring. The emitter is grounded through a $0.11 \mathrm{M} \Omega$ resistor $R$, and the gate and focusing lens electrodes are grounded through a voltage source at voltages $V_{\mathrm{g}}$ and $V_{\mathrm{f}}$, respectively. The electron field emissions occur by applying $V_{\mathrm{g}}$ to the gate electrode around the emitter. The emitted electrons are accelerated toward the anode electrodes along the $z$ direction by $V_{\mathrm{a}}$. We next describe how the electrons are supplied to the $\mathrm{Si}$ emitter tips. Figure 4 shows schematic diagrams of the energy band for (a) $n-\mathrm{Si} / \mathrm{SiO}_{2}$ and (b) $\mathrm{p}-\mathrm{Si} / \mathrm{SiO}_{2}$ regions under the gate electrode. For an n-type $\mathrm{Si}$ emitter tip, electrons are majority carriers. A large value of $V_{\mathrm{g}}$ induces an accumulation of electrons at the $\mathrm{n}-\mathrm{Si} / \mathrm{SiO}_{2}$ interface, and they are supplied to the n-Si emitter, resulting in an electron field emission because of a strong electric field around the emitter tip. In contrast, electrons are minority carriers in the p-type $\mathrm{Si}$ emitter tip. The large value of $V_{\mathrm{g}}$ induces an inversion region at the $\mathrm{p}-\mathrm{Si} / \mathrm{SiO}_{2}$ interface. Electrons thermally excited from the valence band to the conduction band accumulate there, and are supplied to the $\mathrm{p}$-Si emitter, giving rise to an electron field emission. In both cases, the area of cross-shaped gate electrodes is sufficiently large to supply electrons to the emitter tip.

Figure 5(a) shows the EB profile obtained on the anode at $V_{\mathrm{g}}=65 \mathrm{~V}$ and $V_{\mathrm{f}}=20 \mathrm{~V}$. By decreasing $V_{\mathrm{f}}$ from 20 to $12 \mathrm{~V}$ while holding $V_{\mathrm{g}}$ unchanged, the $\mathrm{EB}$ converges into a 
cross-shaped structure [Fig. 5(b)]. The observed EB profile was in agreement with the simulation result obtained using a program (SIMION-3D). ${ }^{36)}$ As described later, the VMS is actually used in this converged configuration to detect the magnetic flux.

Figure 6(a) and (b) shows the $I_{\mathrm{a}}-V_{\mathrm{g}}$ characteristics of the fabricated n-type $\mathrm{Si}$ and the p-type emitter tips, respectively. In the measurement, the gate and in-plane focusing lens electrodes were biased to the same voltage and varied from 0 to $60 \mathrm{~V}$. The emission current appears at around $V_{\mathrm{g}}=40$ and $60 \mathrm{~V}$, respectively, and increases nonlinearly with $V_{\mathrm{g}}$. The tunneling current $I_{\mathrm{FN}}$ generated by the Fowler-Nordheim $(\mathrm{FN})$ mechanism is given by ${ }^{37)}$

$$
I_{\mathrm{FN}}=C_{1} V^{2} \exp \left(-C_{2} / V\right) .
$$

Here, $C_{1}$ and $C_{2}$ are constants, and $V$ is the applied bias voltage, corresponding to $V_{\mathrm{g}}$ in this work. Indicated by open circles in Fig. 6 , the $\log \left(I_{\mathrm{a}} / V_{\mathrm{g}}^{2}\right)$ versus $1000 / V_{\mathrm{g}}$ plot is linear, meaning that the field emission is governed by the FN mechanism. The stability of the emitted current was measured first without focusing. For the n-type $\mathrm{Si}$ emitter tip, $V_{\mathrm{g}}$ and $V_{\mathrm{f}}$ were set to the same value of $45 \mathrm{~V}$. With a further increase in $V_{\mathrm{g}}$, insulation breakdown between the emitter and gate electrodes sometimes happens. The measurement result is shown in Fig. 7(a). The anode current $I_{\mathrm{a}}$ fluctuates by approximately $63 \mathrm{nA}$. Considering that the mean value of $I_{\mathrm{a}}$ was approximately $180 \mathrm{nA}$, the fluctuation in $I_{\mathrm{a}}(\gamma)$ was found to be approximately $\gamma= \pm \frac{63}{2}(\mathrm{nA}) \div 180(\mathrm{nA}) \approx \pm 18 \%$. We next formed the cross-shaped EB by setting the $V_{\mathrm{f}}$ at $12 \mathrm{~V}$; the result is shown in Fig. 7(b). The mean value of $I_{\mathrm{a}}$ was approximately $110 \mathrm{nA}$, and $I_{\mathrm{a}}$ fluctuates up to approximately $110 \mathrm{nA}$, corresponding to $\gamma \approx \pm 50 \%$. Figure 7 (c) shows the time evolution in snap shots of the EB profile on the phosphor. The EB intensity was found to fluctuate as evident in Fig. 7(b). Next, we investigated the EB stability of p-type Si emitter tips. Both $V_{\mathrm{g}}$ and $V_{\mathrm{f}}$ were set to $65 \mathrm{~V}$ so that the measured $I_{\mathrm{a}}$ became almost the same as that in the $\mathrm{n}$-Si emitter tip. The fluctuation in the emitted current was approximately $27 \mathrm{nA}$, significantly decreasing to $\gamma \approx \pm 10 \%$ in Fig. 8(a). Next, the stability of the emitted current was measured when the EB was cross-shaped at $V_{\mathrm{g}}=65 \mathrm{~V}$ and $V_{\mathrm{f}}=10 \mathrm{~V}$. From Fig. 8(b), the fluctuation in $I_{\mathrm{a}}$ was further reduced to $9 \mathrm{nA}$, leading to $\gamma \approx \pm 5 \%$. A constant current source, such as a field effect transistor, to the tips in series is an effective means to reduce these instabilities. $^{38)}$

Many studies have been carried out on current instability in an n-type Si emitter tip. ${ }^{39-41)}$ Hence, in this work, we do not go into this issue but rather focus on the current stability of a 
p-type Si VMS when a magnetic flux is applied.

\subsection{Stability of VMS using a p-type Si emitter tip}

Stability of the VMS using a p-type Si emitter tip was investigated at $V_{\mathrm{g}}=65 \mathrm{~V}$ and $V_{\mathrm{f}}=10 \mathrm{~V}$. We first studied the stability against $B_{z}$. Figure 9(a) shows images of the cross-shaped EB spot rotated by the magnetic flux $B_{\mathrm{z}}$ along the $z$ direction. The cross-shaped EB rotates in a counterclockwise direction in a manner exactly defined by the Lorentz force interaction. The rotation angle $\Delta \Phi$ was measured [Fig. 9(b)]. The solid line shows the calculation result using SIMION-3D. ${ }^{36)}$ Displacement $\Delta \Phi$ increases linearly with $B_{\mathrm{z}}$, and the increase rate is approximately $7 \mathrm{deg} / \mathrm{mT}$, leading to the differential anode current $\Delta I_{\mathrm{a}} / I_{\mathrm{a}}$. Figure 9 (c) shows the dependence of $\Delta I_{\mathrm{a}} / I_{\mathrm{a}}$ on $B_{\mathrm{z}}$. This result means that the rotational displacement can be detected with a sensitivity of approximately $1 \% / \mathrm{mT}$. We next investigated the stability of $\Delta \Phi$ at a fixed value of $B_{\mathrm{z}}$. Figure 9(d) shows images of the cross-shaped EB spot at $B_{\mathrm{Z}}=3.0 \mathrm{mT}$. As presented in Fig. 9(e), the value of $\Delta \Phi$ is fairly stable, and the fluctuation is within $1 \%$. The value of $\Delta \Phi$ decreases linearly with increasing $V_{\mathrm{a}}$ in Fig. 9(f) in accordance with calculations (solid lines). The increase in $V_{\mathrm{a}}$ decreases the transit time for electrons travelling from the emitter tip to the anode, and thus the duration for the Lorentz force to act on the electrons is shortened, resulting in a decrease in $\Delta \Phi$. Regarding the dynamic range of detectable $B_{z}$, the measured difference in anode current $\left(\Delta I_{\mathrm{a}} / I_{\mathrm{a}}\right)$ changes periodically against $B_{z}$ on the basis of the working principle of the VMS, making us difficult to determine the value of $B_{z}$. In such a case, we can detect higher $B_{z}$ by setting $V_{\text {a }}$ to be a smaller value to suppress the velocity of electrons along the $z$ direction $\left(v_{z}\right)$ as presented in Fig. 9(f).

Figure 10 shows the time evolution of $\Delta \Phi$ obtained for VMS using an n-type Si emitter tip at $B_{\mathrm{z}}=3.0 \mathrm{mT}, V_{\mathrm{g}}=45 \mathrm{~V}, V_{\mathrm{f}}=12 \mathrm{~V}$, and $V_{\mathrm{a}}=700 \mathrm{~V}$. Compared to $\Delta \Phi$ being fairly stable for p-type Si VSM as shown in Fig. 9(e), the fluctuation in $\Delta \Phi$ reaches approximately $5 \%$ for n-type Si VMS. This value is much higher than that for the p-type Si VMS shown in Fig. 9(e) as anticipated from Fig. 7(b).

We next examined the stability against $B_{x}$. The $B_{x}$ gives rise to the translational displacement of the cross-shaped EB in the $y$ direction $\left(\Delta L_{y}\right)$ by the Lorentz force $\left(F_{\mathrm{y}}\right)$ acting on the $\mathrm{EB}\left(F_{y}=-e v_{z} \times B_{x}\right)$ and thereby induces $\Delta I_{\mathrm{a}} / I_{\mathrm{a}}$. The transit time $t_{0}$ necessary for emitted electrons to reach the anode is given by, 


$$
t_{0}=d \sqrt{\frac{2 m_{0}}{e V_{a}}} .
$$

Therefore, $\Delta L_{y}$ is presented by,

$$
\Delta L_{y}=\left|\int_{0}^{t_{0}} v_{y} d t\right|=\sqrt{\frac{8 e}{9 m_{0} V_{a}}} d^{2} B_{x} .
$$

Here, $e$ is the elemental charge, $m_{0}$ is the electron rest mass in vacuum. Figure 11(a) shows images of the EB spot when increasing $B_{\mathrm{x}}$. The measured $\Delta L_{y}$ from the initial position was plotted [Fig. 11(b)] together with the calculated results marked by a solid line. $\Delta L_{y}$ increases linearly with $B_{\mathrm{x}}$, resulting in an increase in $\Delta I_{\mathrm{a}} / I_{\mathrm{a}}$ [Fig. 11(c)]. The characteristics shows good linearity and a sensitivity as high as $10 \% / \mathrm{mT}$. Figure $11(\mathrm{~d})$ shows the time evolution of $\Delta L_{y}$ at $B_{\mathrm{x}}=3.0 \mathrm{mT}$ over a $90 \mathrm{~s}$ duration, demonstrating that $\Delta L_{y}$ is quite stable. On the basis of these results, we can state that the three-axis components of an external $B$ applied in an arbitrary direction is detectable with high sensitivity and stability using a cross-shaped EB from the p-type Si emitter tip.

\section{Conclusion}

The Si field emitter tips were fabricated using a conventional fabrication process under photolithography and RIE, and their emission current stability was investigated in vacuum. The field emission of the current was well explained by the FN mechanism for both p- and n-type Si emitter tips. The p-Si emitter tip was much more stable than the n-type Si emitter tip. In the p-Si emitter tip, the magnetic flux parallel to the $\mathrm{EB}, B_{\mathrm{z}}$, was detected by the rotational displacement of the cross-shaped EB, and sensed as a differential anode current $\Delta I_{\mathrm{a}} / I_{\mathrm{a}}$. The sensitivity reached approximately $1 \% / \mathrm{mT}$. The value of $\Delta \Phi$ was fairly sensitive to $B_{\mathrm{z}}$ and stable. The fluctuation in $\Delta \Phi$ was within $1 \%$ at $B_{\mathrm{z}}=3.0 \mathrm{mT}$. The magnetic flux normal to the $\mathrm{EB}, B_{\mathrm{x}}$, was detected by the translational displacement $\Delta L_{y}$ and sensed as $\Delta I_{\mathrm{a}} / I_{\mathrm{a}}$ with greater sensitivity as high as $10 \% / \mathrm{mT}$. The fluctuation in $\Delta L_{y}$ was also within $1 \%$ at $B_{\mathrm{z}}=3.0 \mathrm{mT}$.

These results demonstrate that the VMS with a vertical p-Si emitter tip exploiting a cross-shaped EB is able to detect an arbitrarily directed magnetic flux with high sensitivity.

\section{Acknowledgment}

The authors thank Dr. J. Itoh and Dr. S. Kanemaru for their helpful advice. 


\section{References}

1. C. A. Spindt, J. Appl. Phys. 39, 3504(1968).

2. C. A. Spindt, C. E. Holland, A. Rosengreen, and I. Brodie, IEEE Trans. Electron Devices 38, 2355 (1991).

3. S. Itoh, T. Niiyama, M. Taniguchi, and T. Watanabe, J. Vac. Sci. Technol. B14, 1977 (1996).

4. T. Utsumi, IEEE Trans. Electron Devices 38, 2276 (1991).

5. H. Gamo, S. Kanemaru, and J. Itoh, Jpn. J. Appl. Phys. 34, 6916 (1995).

6. M. Urayama, T. Ise, Y. Maruo, A. Kishi, R. Imamoto, and T. Takase, Jpn. J. Appl. Phys. 32, 6293 (1993).

7. J. Itoh, Proc. $1^{\text {st }}$ Int. Display Workshops 11 (1994)

8. Y. Toma, S. Kanemaru, and J. Itoh, J. Vac. Sci. Technol. B14, 1902 (1996).

9. Y. F. Zakharchenko, G. V. Torgashov, Y. V. Gulyaev, N. I. Sinitsyn, I. S. Nefedov, A. I. Zhbanov, and E. M. II'in, J. Vac. Sci. Technol. B14, 1982 (1996).

10. C. A. Spindt, C. E. Holland, P. Fl. Schwoebel, and 1. Brodie, J. Vac. Sci. Technol. B14, 1986 (1996).

11. C. M. Tang, M. Goldstein, T. A. Swyden, J. E. Walsh, Nucl. Instrum. Methods A358, 7 (1995).

12. I. Honjo, Y. Endo, and S. Gotobi, J. Vac. Sci. Technol. B15, 2742 (1997).

13. Y. Endo, I. Honjo, and S. Gotobl, J. Vac. Sci. Technol. B16, 3082 (1998).

14. R. Baptist, C. Bieth and C. Py, J. Vac. Sci. Technol. B14, 2119 (1996).

15. D. K. Schroder, R. N. Thomas, J. Vine, and H. C. Nathanson, IEEE Trans. Electron Devices 21, 785 (1974).

16. R. N. Thomas, R. A. Wickstrom, D. K. Schroder and H. C. Nathanson, Solid-Stale Electron 17, 155 (1974).

17. A. Aboubacar, A. Chbihi, M. Dupom, J Gardes, M. Laguna, and M. Querrou, J. Vac. Sci. Technol. B13, 616 (1995).

18. S. Mingels, V. Porshyn, C. Prommesberger, C. Langer, R. Shreiner, D. Luetzenkirchen-Hecht, and G. Muller, J. Appl. Phys. 119, 165104 (2016).

19. L. F. Velasquez-Garcia, S. A. Guerrera, Y. Niu, and Al. Akinwande, IEEE Trans. Electron Devices 58, 1775 (2011).

20. L. F. Velasquez-Garcia, S. A. Guerrera, Y. Niu, and Al. Akinwande, IEEE Trans. Electron Devices 58, 1783 (2011).

21. H. E. Burke, in Handbook of Magnetic Phenomena (Springer, 1986) p. 378.

22. A. Nathan, A. M. J. Huiser, and H. P. Baltes, IEEE Trans. Electron Devices 32, 1212 (1935).

23. Y. Sugiyama, H. Soga, M. Tacano, and Henry P. Baltes, IEEE Trans. Electron Devices 36, 1639 (1989).

24. V. Zieren and B.P.M. Duyndam, IEEE Trans. Electron Devices 29, 83 (1982).

25. J. Itoh, K. Tsuburaya. S. Kanemaru, T. Watanabe, and S. Itoh, Jpn. J. Appl. Phys. 31, L884 (1992).

26. J. Itoh, K. Tsuburaya, S. Kanemaru, T. Watanabe, and S. Itoh, Jpn. J. Appl. Phys. 32, 1221 (1993).

27. J. Itoh, K. Tsuburaya, and S. Kanemaru, Tech. Dig. of the 11th Sensor Symp. 143 (1992).

28. Y. Sugiyama, J. Itoh, and S. Kanemaru, Proc. $7^{\text {th }}$ Int. Conf. Solid-State-Sensors and actuators 884, Yokohama, Japan, (1993).

29. K. Uemura, S. Kanemaru, and J. Itoh, Jpn. J. Appl. Phys. 35, 6629 (1996).

30. W. B. Herrmannsfeldt, R. Becker, I. Brodie, A. Rosengreen, and C. A. Spindt, Nuclear Instru. \& Method in Phys. Res. A 298, 39 (1990).

31. J. Itoh, K. Uemura, S. Kanemaru, J. Vac. Sci. Technol. B16, 1233 (1998).

32. J. ltoh, Y. Tohma, K. Morikawa, S. Kanemaru, and K. Shimizu, J. Vac. Sci. Technol. B13, 1968 (1995).

33. K. Uemura, S. Kanemaru, and J. Itoh, Jpn. J. Appl. Phys. 36, 7754 (1997).

34. K. Uemura, S. Kanemaru, and J. Itoh, J. Micromech. Microeng. 11, 81 (2001).

35. K. Betsui, IVMC Tech. Dig., 1991, p. 26.

36. D. A. Dahl, Simion 3D Version 6.0, Idaho National Engineering Laboratory, Chemical Materials \&

Processing Department, Lockheed Idaho Technologies Company, Idaho Falls, ID 83415.

37. S. M. Sze, Physics of Semiconductor Devices, $2^{\text {nd }}$ ed. (Wiley, New York, 1981), Chap. 7.

38. T. Hirano, S. Kanemaru, H. Tanoue, and J. Itoh, Jpn. J. Appl. Phys. 35, 6637 (1996).

39. K. Sawada, K. Ji, and T. Ando, Jpn. J. Appl. Phys. 33, L1345 (1994).

40. J. Itoh and S. Kanemaru, Hyomen Kagaku 17, 718 (1996. (in Japanese). 
41. Wei Zhu, Vacuum Microelectronics, $1^{\text {st }}$ ed. (John Wiley \& Sons), Chap. 5. 
Fig. 1 (a) SEM image, and schematics of the (b) emitter, (c) anode electrodes, and (d) whole device structure.

Fig. 2 Fabrication process flow of VMS.

Fig. 3 Schematic of measurement system. $V_{\mathrm{g}}, V_{\mathrm{f}}$, and $V_{\mathrm{a}}$ are voltages applied to gate, focusing lenses, and anode electrodes with respect to the ground. $I_{e}, I_{\mathrm{g}}, I_{\mathrm{f}}, I_{\mathrm{a} 1}, I_{\mathrm{a} 2}$ are currents. $R$ is the resistance.

Fig. 4 Schematic band diagrams of (a) n-Si/SiO 2 and (b) p-Si/ $\mathrm{SiO}_{2}$ interfaces under the gate electrode along the $z$ direction under bias voltages.

Fig. 5 EB profiles on the phosphor-covered anode obtained at (a) $V_{\mathrm{f}}=20 \mathrm{~V}$ and (b) $V_{\mathrm{f}}=12 \mathrm{~V}$ when $V_{\mathrm{g}}=50 \mathrm{~V}$ and $V_{\mathrm{a}}=700 \mathrm{~V}$. Simulation result is also shown in (b).

Fig. 6 Emission current $I_{\mathrm{a}}$ versus gate voltage $V_{\mathrm{g}}$ characteristics for (a) n-type and (b) p-type Si emitter tips under the condition $V_{\mathrm{g}}=V_{\mathrm{f}}$.

Fig. 7 Emission current stability of the n-Si emitter tip under (a) non-convergent condition at $V_{\mathrm{g}}=V_{\mathrm{f}}=45 \mathrm{~V}$, (b) convergent condition at $V_{\mathrm{g}}=45 \mathrm{~V}, V_{\mathrm{f}}=12 \mathrm{~V}$ when $V_{\mathrm{a}}=700 \mathrm{~V}$. (c) Time evolution of the EB pattern on the phosphor-covered anode screen in condition (b).

Fig. 8 Emission current stability of the p-Si emitter tip under (a) non-convergent condition at $V_{\mathrm{g}}=V_{\mathrm{f}}=65 \mathrm{~V}$, (b) convergent condition at $V_{\mathrm{g}}=65 \mathrm{~V}, V_{\mathrm{f}}=10 \mathrm{~V}$ when $V_{\mathrm{a}}=700 \mathrm{~V}$.

Fig. 9 (a) Cross-shaped EB profiles at different values of $B_{\mathrm{z}}$ at $V_{\mathrm{g}}=65 \mathrm{~V}, V_{\mathrm{f}}=10 \mathrm{~V}$, and $V_{\mathrm{a}}=700 \mathrm{~V}$. Dependences of (b) rotational angle $\Delta \Phi$ and (c) differential anode current $\Delta I_{\mathrm{a}} / I_{\mathrm{a}}$ on $B_{\mathrm{z}}$ under the same condition in (a). ${ }^{28)}$ Time evolutions of (d) EB profiles on the phosphor screen and (e) $\Delta \Phi$ at $B_{\mathrm{z}}=3.0 \mathrm{mT}$ under the same condition in (a). (f) Relationship between $\Delta \Phi$ and anode bias voltage $V_{\mathrm{a}}$ at $B_{\mathrm{z}}=2.0 \mathrm{mT}$ when $V_{\mathrm{g}}=65 \mathrm{~V}$ and $V_{\mathrm{f}}=$ $10 \mathrm{~V}$.

Fig. 10 Time evolution of $\Delta \Phi$ obtained for VMS using an n-type Si emitter tip at $B_{\mathrm{z}}=3.0 \mathrm{mT}, V_{\mathrm{g}}=45 \mathrm{~V}, V_{\mathrm{f}}=$ $12 \mathrm{~V}$, and $V_{\mathrm{a}}=700 \mathrm{~V}$.

Fig. 11 (a) Dependence of the translational displacement of the EB spot $\Delta L_{y}$ along the $y$ direction for various values of $B_{\mathrm{x}}$. Dependences of (b) $\Delta L_{y}$ and (c) $\Delta I_{\mathrm{a}} / I_{\mathrm{a}}$ on $B_{\mathrm{x}}{ }^{26}$ ) (d) Stability of $\Delta L_{y}$ at $B_{\mathrm{x}}=3.0 \mathrm{mT}$. All measurements were performed at $V_{\mathrm{g}}=65 \mathrm{~V}, V_{\mathrm{f}}=10 \mathrm{~V}$, and $V_{\mathrm{a}}=700 \mathrm{~V}$. 
(a)

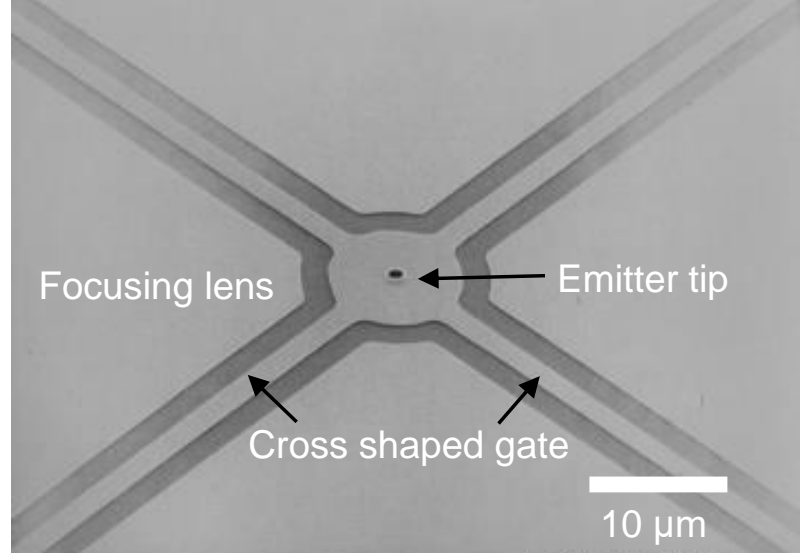

(b)

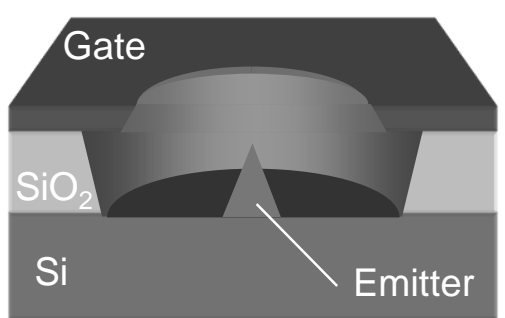

(c)

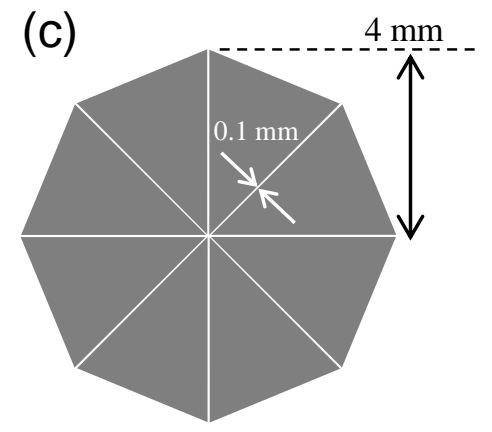

(d)

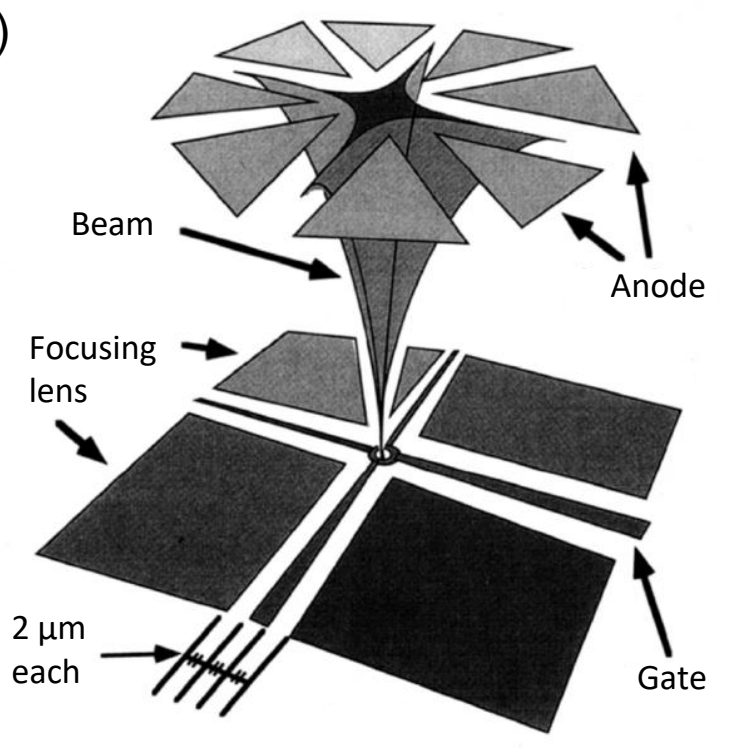

Fig. 1 


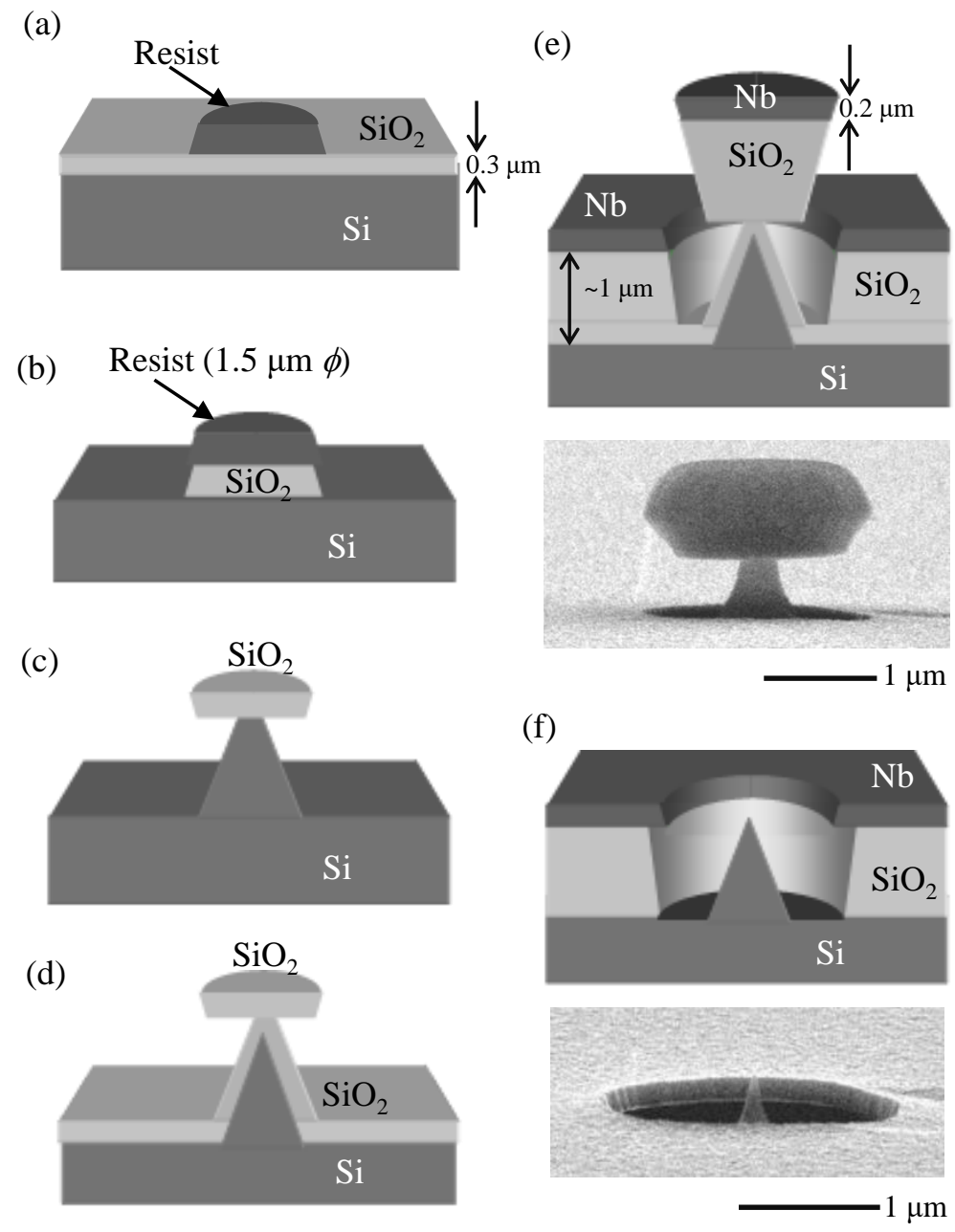

Fig. 2 


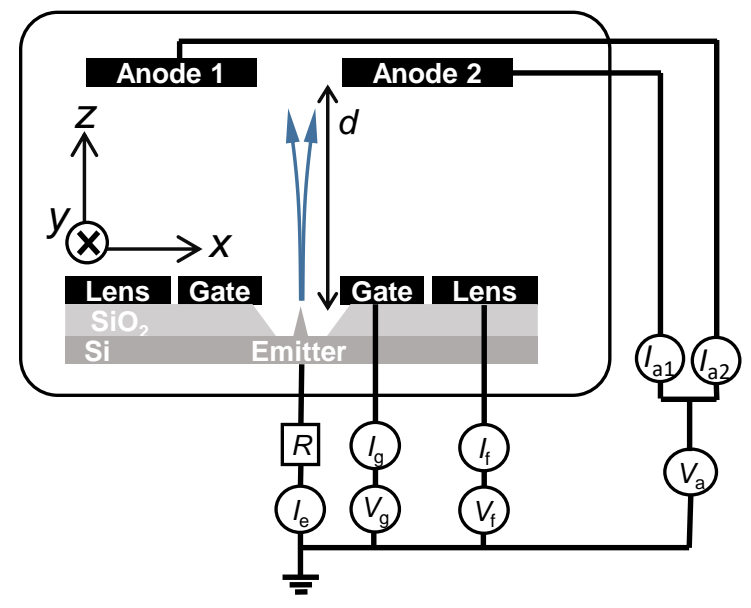

Fig. 3 


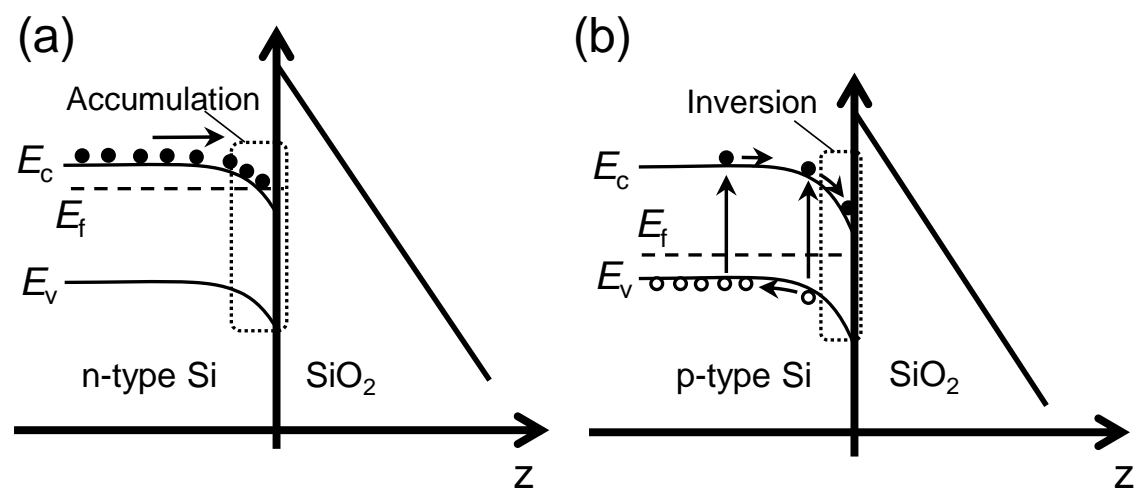

Fig. 4 
(a)

(b)
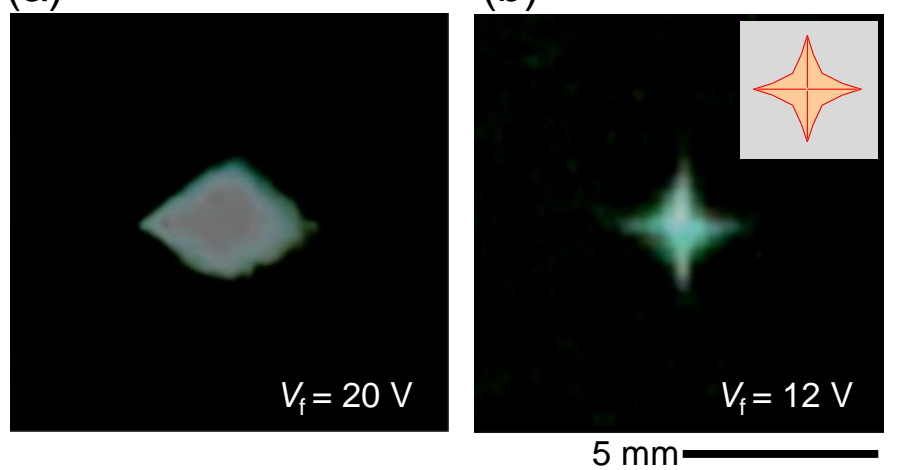

Fig. 5 

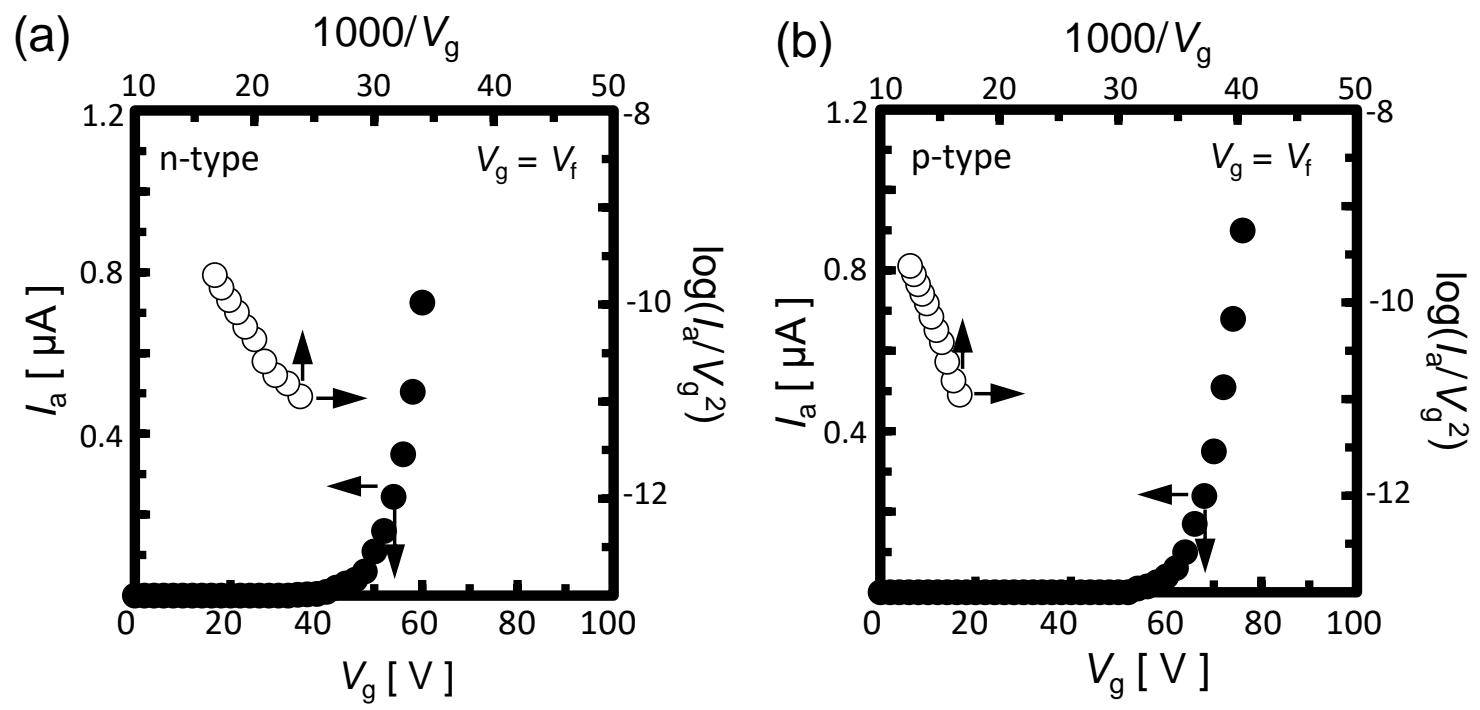

Fig. 6 
(a)
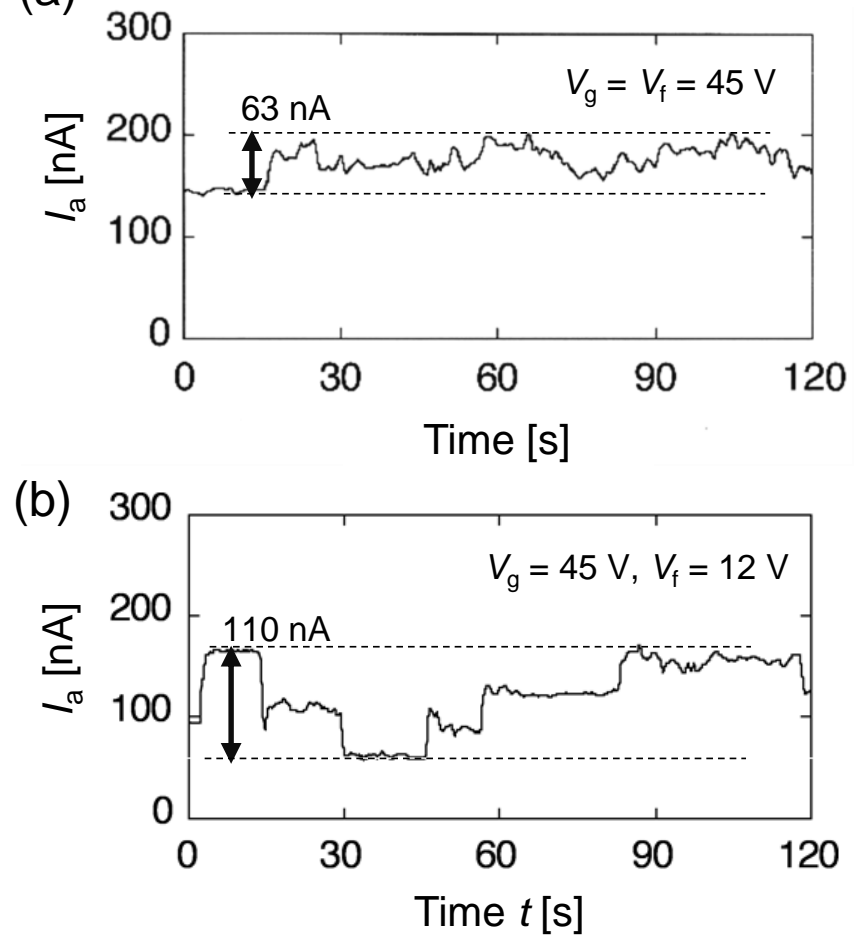

(c)
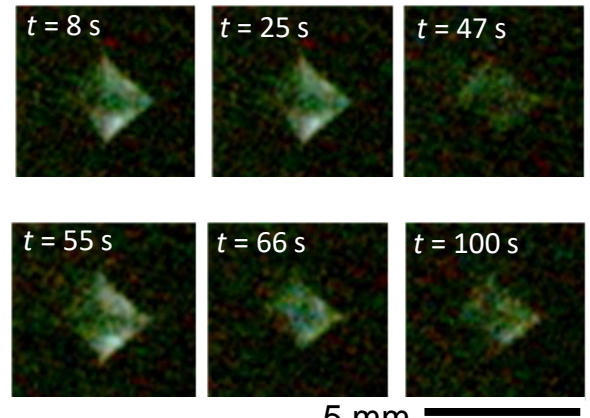

Fig. 7 
(a)
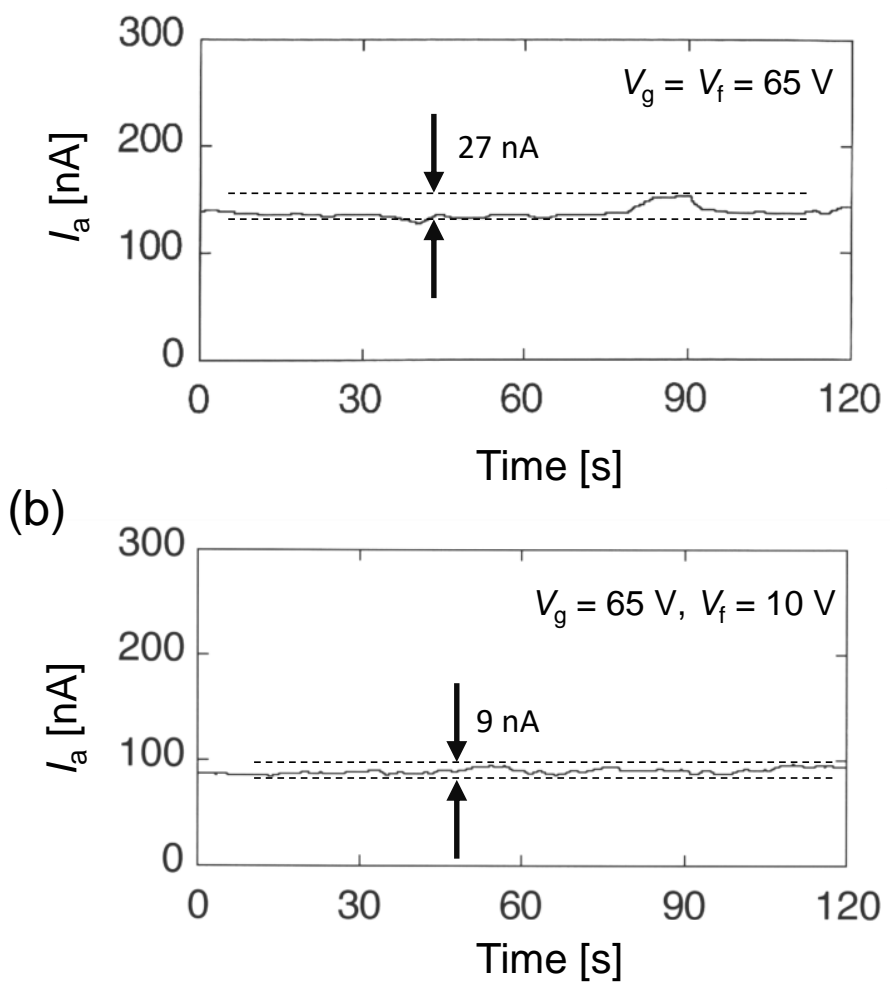

Fig. 8 
(a)

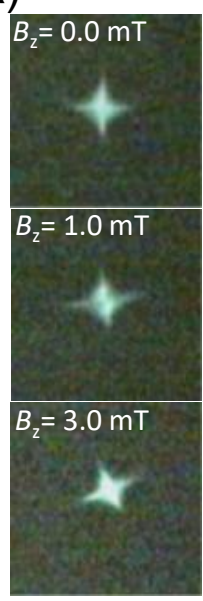

(d)

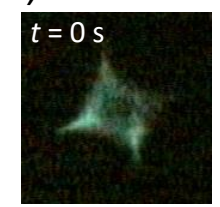

(e)
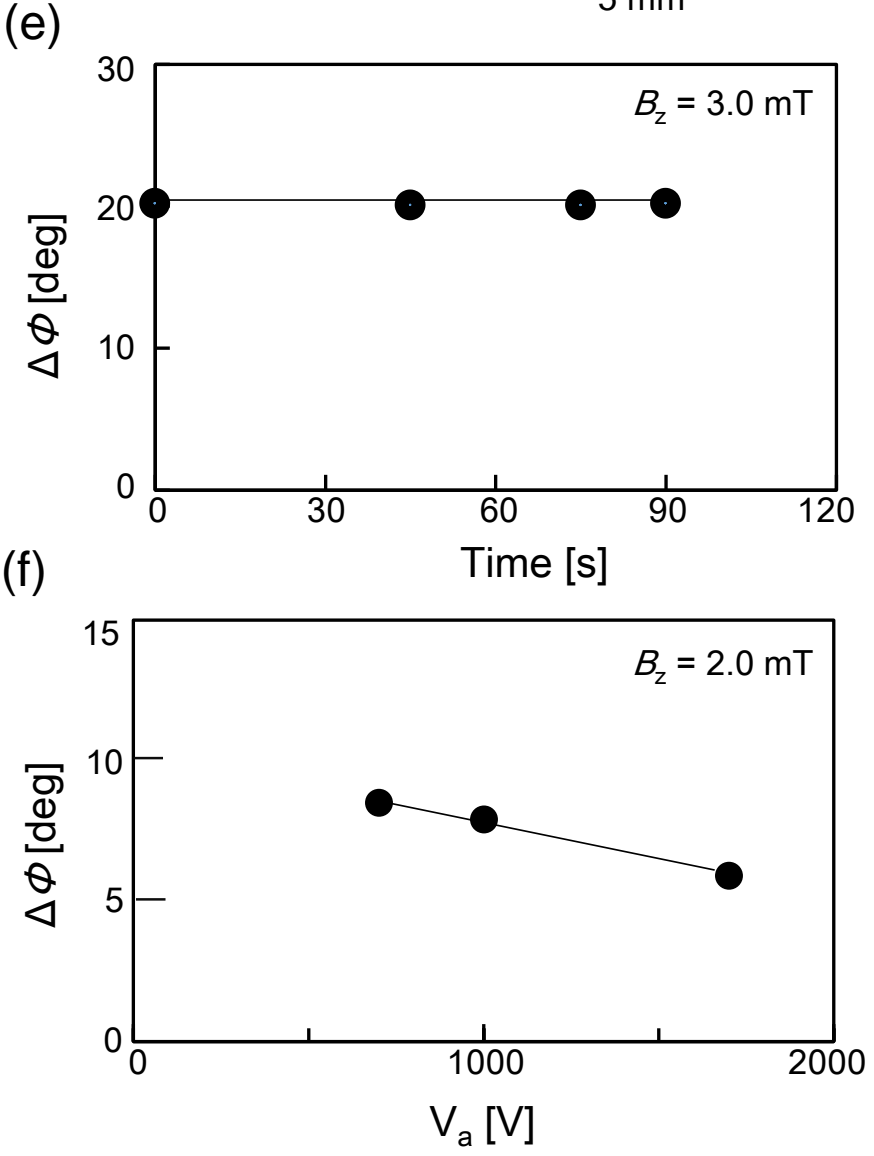

(b)

(c)

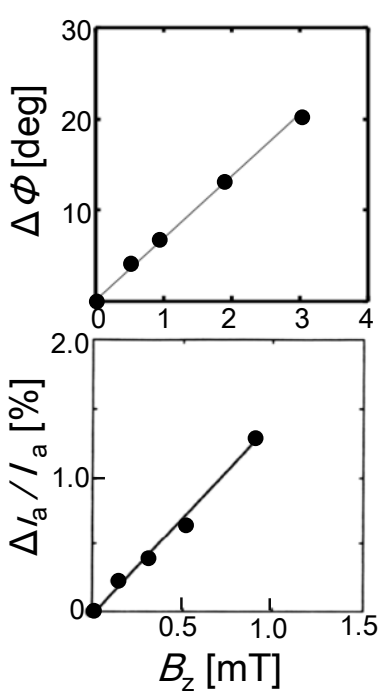

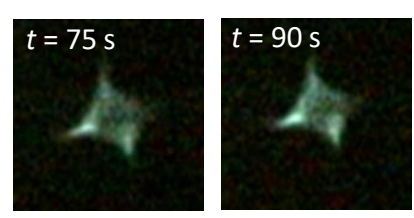

$5 \mathrm{~mm}$

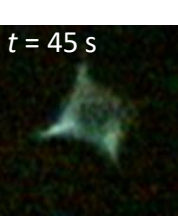




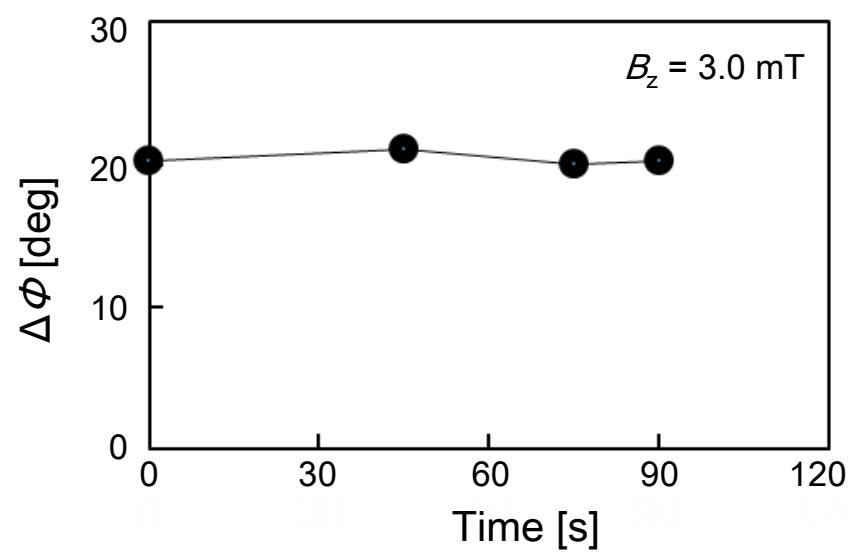

Fig. 10 
(a)

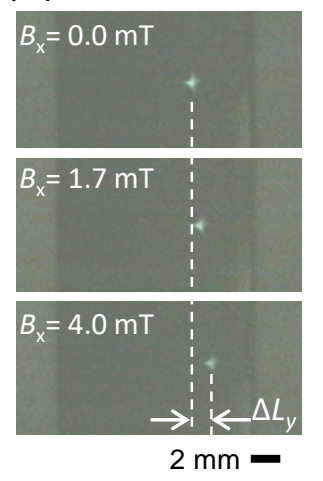

(d)

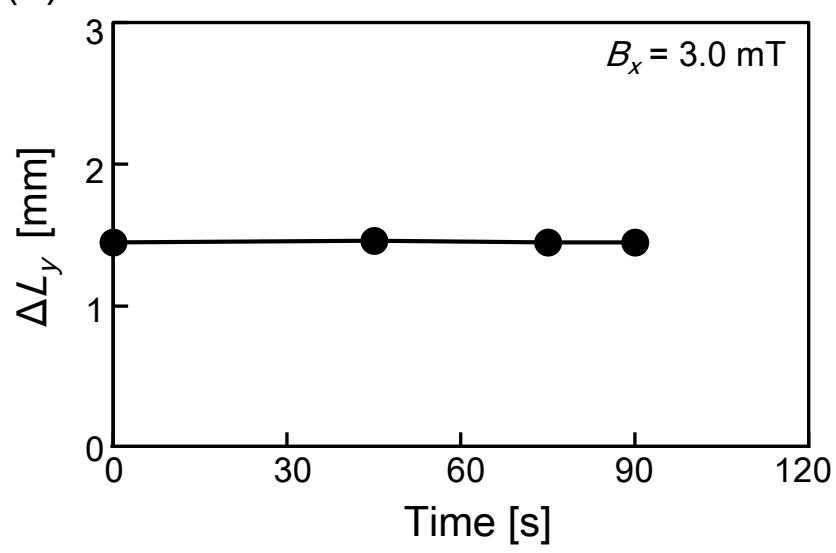

(b)

(c)

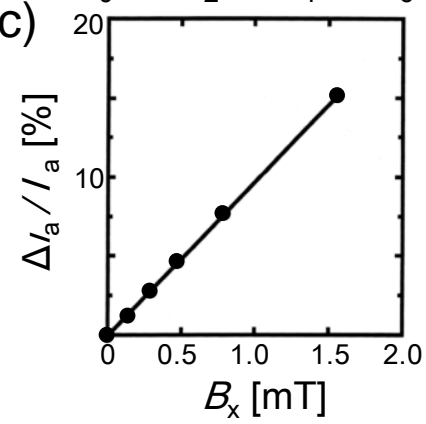

Fig. 11 